\title{
Estimação da digestibilidade do extrato etéreo em ruminantes a partir dos teores dietéticos: desenvolvimento de um modelo para condições brasileiras
}

\author{
Edenio Detmann ${ }^{1}$, Sebastião de Campos Valadares Filho ${ }^{1}$, Douglas dos Santos Pina ${ }^{2}$, \\ José Maurício de Souza Campos ${ }^{1}$, Mário Fonseca Paulino ${ }^{1}$, André Soares de Oliveira², \\ Polyana Albino Silva ${ }^{2}$
}

\footnotetext{
${ }^{1}$ Departamento de Zootecnia, Universidade Federal de Viçosa, Viçosa-MG, 36571-000, Bolsista do CNPq.

2 Doutorando em Zootecnia - UFV.
}

RESUMO - Objetivou-se neste estudo propor e validar um modelo para estimação da fração aparentemente digestível do EE a partir dos teores dietéticos em bovinos. As bases teóricas para o desenvolvimento do modelo fundamentaram-se no coeficiente de digestibilidade verdadeiro e na contribuição metabólica fecal. O método de estimação destes parâmetros baseou-se no teste de entidade nutricional. O banco de dados utilizado para construção do modelo foi constituído por 48 e 60 médias experimentais para vacas em lactação e bovinos em crescimento e terminação, respectivamente, tomadas de 24 experimentos, 12 para cada categoria animal. Adotou-se o esquema de identidade de modelos por variável Dummy para diferenciação entre categorias animais. O banco de dados utilizado para validação do modelo proposto foi obtido de três experimentos, sendo dois com vacas em lactação e um com bovinos em crescimento. O coeficiente de digestibilidade verdadeiro do EE não diferiu entre categorias animais, cuja estimativa foi de 0,8596. Verificou-se superioridade da fração metabólica fecal para vacas em lactação. Essa porção mostrou-se não relacionada ao teor de EE (EE\%) na dieta, quando expressa como percentual dietético, para ambas as categorias. Assim, para animais em condições de alimentação ad libitum, o teor de EE aparentemente digestível (EE ${ }_{a d} \%$ ) pode ser estimado pelas equações: $\mathrm{EE}_{\mathrm{ad}} \%=0,8596 \mathrm{EE} \%-0,21$, para vacas em lactação; e $\mathrm{EE}_{\mathrm{ad}} \%=0,8596 \mathrm{EE} \%-0,18$, para bovinos em crescimento e terminação. O processo de validação indicou similaridade entre valores preditos e observados de $\mathrm{EE}_{\mathrm{ad}} \%$ para ambas as categorias animais. Conclui-se que os modelos propostos poderão ampliar a acurácia de predição do teor de nutrientes digestíveis totais a partir da composição química da dieta.

Palavras-chave: digestibilidade aparente, sistema NRC, teste de entidade nutricional

\section{Estimation of ether extract digestibility in diets of ruminants: a model under Brazilian conditions}

\begin{abstract}
The objective of this study was to develop and validate a model to estimate the EE apparently digestible fraction from different cattle diets. True digestibility coefficients and metabolic fecal contribution were the main parameters fitted to the model. The estimation method for both parameters was based on the Lucas test. The dataset used to build the model contained 48 and 60 experimental means for lactating cows and growing and finishing cattle, respectively. Data were taken from 24 experiments (12 experiments for each animal category). Comparison between animal categories was done by using a Dummy variable. The dataset used for model validation originated from two independent trials with lactating cows and one trial with growing cattle. The true digestibility coefficient $(0.8596)$ did not differ between animal categories. It was observed greater metabolic fecal EE for lactating cows than for growing and finishing cattle. The metabolic fecal EE did not change with different dietary EE levels (EE\%), when expressed as a percentage of dietary EE. Therefore, the EE apparently digestible in the diet $\left(\mathrm{EE}_{\mathrm{ad}} \%\right)$ can be estimated by the following equations: $\mathrm{EE}_{\mathrm{ad}} \%=0.8596 \mathrm{EE} \%-0.21$ for lactating cows and $\mathrm{EE}_{\mathrm{ad}} \%=0.8596 \mathrm{EE} \%-0.18$ for growing and finishing cattle. The validation procedure indicated that observed and estimated values of $\mathrm{EE}_{\mathrm{ad}} \%$ were not different for both animal categories. It was concluded that the proposed model can improve the accuracy of total digestible nutrients prediction based on chemical composition of the diet.
\end{abstract}

Key Words: apparent digestibility, Lucas test, NRC system

\section{Introdução}

A estimação dos parâmetros de digestibilidade de um alimento constitui aspecto preponderante ao acesso ao seu teor energético, notadamente via nutrientes digestíveis totais, permitindo o balanceamento adequado de dietas que propiciem o atendimento das demandas para mantença e produção dos animais. 
No entanto, mesmo que consista em um parâmetro digestivo estático, ou seja, que pode ser representado por uma estimativa pontual, o acesso à digestibilidade de um alimento ou de seus respectivos componentes químicos é oneroso e demanda muito tempo para obtenção de estimativas quando realizado pelos métodos clássicos in vivo.

Embora métodos alternativos para estimação de parâmetros da digestibilidade tenham sido desenvolvidos em ambientes in situ ou in vitro, tanto por vias gravimétricas (Tilley \& Terry, 1963) quanto metabólicas (Schofield et al., 1994), as estimativas têm apresentado vícios substanciais em relação aos valores observados in vivo para alimentos produzidos em condições tropicais (Vieira et al., 2000; Detmann et al., 2005).

De forma alternativa aos métodos biológicos, a utilização da composição química dos alimentos como método de predição da digestão em ruminantes tem sido sugerida por diversos autores (Conrad et al, 1984; Weiss et al., 1992; Van Soest, 1994). Diante dessa perspectiva, o NRC (2001) propôs a adoção de um método com esse embasamento para a estimação do teor de NDT dos alimentos que constitui um sistema de equações somativas no qual a cada grupo de compostos químicos destina-se uma equação para obtenção de estimativas de coeficientes de digestibilidade verdadeiros, com posteriores correções para as perdas metabólicas e o nível de consumo.

Contudo, embora sendo teoricamente embasado (Conrad et al., 1984; Weiss et al., 1992), esse sistema tem apresentado eficiência de predição não satisfatória quando aplicado a alimentos obtidos em condições tropicais (Rocha Jr. et al., 2003; Campos, 2004; Detmann et al., 2004; Oliveira, 2005), tornando as estimativas substancialmente desviadas dos valores observados in vivo.

De forma mais específica, por se tratar de um sistema somativo (Weiss et al., 1992; NRC, 2001), as divergências das estimativas de NDT consistem em um somatório simples das divergências observadas para cada grupo de compostos separadamente (EE, FDN, CNF e PB). Desta forma, possíveis correções sobre o sistema somativo adotado pelo NRC (2001) devem ser investigadas quanto às estimativas individuais de cada grupo de compostos, as quais refletirão diretamente sobre a exatidão das estimativas obtidas para o nível de NDT do alimento/dieta.

Embora com concentrações extremamente inferiores aos demais componentes na maioria dos alimentos produzidos em condições tropicais, o EE apresenta posição central na obtenção de estimativas do teor energético, em razão de sua elevada concentração calórica.

Entre os grupos de compostos orgânicos de um alimento, as estimativas dos teores de EE digestíveis obtidas via sistema de equações somativas (NRC, 2001) têm contribuído substancialmente para a falta de exatidão das estimativas de NDT em condições tropicais (Rocha Jr. et al., 2003), incluindo-se valores inverossímeis (Campos, 2004) ou sem correlação com os obtidos in vivo (Oliveira, 2005). Esse quadro suporta a necessidade premente de correção ou substituição da equação relacionada a esse grupo de compostos.

Segundo proposições de Lucas \& Smart (1959), componentes dos alimentos com comportamento digestivo homogêneo, como $\mathrm{EE}$ e $\mathrm{PB}$, poderiam ter suas estimativas de digestibilidade aparente obtidas a partir de seu coeficiente de digestibilidade verdadeiro e de sua contribuição metabólica fecal. Esse processo de estimação passou a ser denominado teste de entidade nutricional (Lucas \& Smart, 1959; Van Soest, 1994) e seu potencial para aplicação em condições tropicais ainda é desconhecido, podendo ser uma alternativa para construção de modelo de maior acurácia para estimação da fração digestível do EE a partir dos teores dietéticos.

Objetivou-se neste estudo desenvolver e validar um modelo para estimação da digestibilidade do EE a partir de suas concentrações dietéticas, com base no coeficiente de digestibilidade verdadeiro e na contribuição metabólica fecal, em animais alimentados em condições tropicais.

\section{Material e Métodos}

A construção do modelo foi realizada utilizando-se um banco de dados constituído por médias de tratamentos obtidas em 12 experimentos com bovinos em crescimento e terminação (Carvalho et al., 1997; Ladeira et al., 1999; Cardoso et al., 2000; Dias et al., 2000; Tibo et al., 2000; Ítavo et al., 2002a; 2002b; Rennó, 2003; Chizzotti et al., 2005) e 12 com vacas em lactação (Malafaia et al., 1996 a, b; Mora et al., 1996 a, b; Moreira et al., 2001; Oliveira et al., 2001; Silva et al., 2001; Assis et al., 2004a; 2004b; Chizzotti, 2004; Magalhães et al., 2004a; 2004b; Mendonça et al., 2004; Nörnberg et al., 2004; Soares et al., 2004).

Foram levantadas informações relativas às variáveis: teor dietético ( $\%$ da MS), consumo (g/dia) e digestibilidade aparente total (\%) do EE. Em todos os experimentos avaliados, verificou-se alimentação ad libitum dos animais e avaliação das concentrações de EE em amostras de alimentos, sobras e fezes pelo método de extração "a quente" em éter (método Goldfish) (AOAC, 1990). As estatísticas descritivas para as variáveis avaliadas são descritas na Tabela 1.

Sob a pressuposição de as médias de tratamentos constituírem estimadores independentes e não-viesados 
Tabela 1 - Estatísticas descritivas para as variáveis nível de EE na dieta (EE, \% - \% da MS), consumo diário de EE (CEE, g)e digestibilidade aparente do EE (DEE, \%)

Table 1 - Descriptive statistics for dietary EE level (EE, \% - \% of DM), EE daily intake (EEI, g), and apparent digestibility of EE (EED, \%)

\begin{tabular}{|c|c|c|c|c|c|c|}
\hline \multirow[b]{2}{*}{$\begin{array}{l}\text { Estatística } \\
\text { Statistic }\end{array}$} & \multicolumn{3}{|c|}{$\begin{array}{l}\text { Vacas em lactação } \\
\text { Lactating cows }\end{array}$} & \multicolumn{3}{|c|}{$\begin{array}{l}\text { Bovinos em crescimento e terminação } \\
\text { Growing and finnishing cattle }\end{array}$} \\
\hline & EE $(\%)$ & $\begin{array}{c}\text { CEE } \\
\text { EEI }\end{array}$ & $\begin{array}{l}\mathrm{DEE} \\
E E D\end{array}$ & $\mathrm{EE}(\%)$ & $\begin{array}{c}\text { CEE } \\
E E I\end{array}$ & $\begin{array}{l}\mathrm{DEE} \\
\text { EED }\end{array}$ \\
\hline Média (Mean) & 2,84 & 491,3 & 74,02 & 2,23 & 132,4 & 77,76 \\
\hline Moda (Mode) & 2,77 & 300,0 & 81,60 & 2,03 & 120,0 & - \\
\hline Mediana (Median) & 2,65 & 451,5 & 81,60 & 2,18 & 130,0 & 80,41 \\
\hline Desvio-padrão (Standard deviation) & 1,54 & 279,7 & 19,39 & 0,52 & 47,0 & 8,36 \\
\hline $\mathrm{n}$ & & 48 & & & 60 & \\
\hline
\end{tabular}

das médias populacionais, os dados levantados foram avaliados por meio de adaptação ao teste de entidade nutricional relatado por Lucas \& Smart (1959), que se baseia no ajustamento de regressão linear simples, segundo o modelo:

$Y_{i}=\beta X_{i}+\alpha+\varepsilon_{i}$

em que: $\mathrm{Y}_{\mathrm{i}}=\mathrm{EE}$ aparentemente retido ou digerido (g/dia); $\mathrm{X}_{\mathrm{i}}=$ EE consumido $(\mathrm{g} / \mathrm{dia}) ; \beta=$ coeficiente de digestibilidade verdadeiro para o EE; $\alpha=$ EE metabólico fecal (g/dia); $\varepsilon_{\mathrm{i}}=$ erro aleatório, pressuposto normal e independentemente distribuído, com média zero e variância $\sigma^{2}$.

Para que o componente químico do alimento/dieta seja considerado entidade nutricional, validando-se, portanto, o processo de estimação, as seguintes restrições devem ser observadas (Lucas \& Smart, 1959):

$0 \leq \hat{\beta} \leq 1$

$\hat{\alpha} \leq 0$

Em razão da possível diferença entre as categorias animais avaliadas, inseriu-se à equação (1) um esquema de avaliação de identidade de modelos por intermédio de variável dummy (Draper \& Smith, 1966). Para estimação do erro puro, introduziu-se controle da variação experimental em função da variabilidade interexperimentos. Desta forma, redefiniu-se o modelo descrito em (1) como:

$Y_{i j k}=\beta_{1} X_{i j k}+\beta_{2} C_{i} X_{i j k}+\alpha_{1}+\alpha_{2} C_{i}+E_{(i) k}+\varepsilon_{i j k}$

em que: $\mathrm{Y}_{\mathrm{ijk}}=\mathrm{EE}$ aparentemente retido obtido na observação j, referente ao experimento $\mathrm{k}$, sobre a categoria animal i (g/dia); $X_{i j k}=$ EE consumido obtido na observação j, referente ao experimento $\mathrm{k}$, sobre a categoria animal $\mathrm{i}$ (g/dia); $\mathrm{C}_{\mathrm{i}}=$ variável dummy referente à categoria animal $\mathrm{i}$, sendo $\mathrm{C}_{1}=0$ para animais em crescimento e terminação e $\mathrm{C}_{2}=1$, para vacas em lactação; $\beta_{1}$ e $\beta_{2}=$ parâmetros para estimação do coeficiente de digestibilidade verdadeiro do EE; $\alpha_{1}$ e $\alpha_{2}=$ parâmetros para estimação do EE metabólico fecal $(\mathrm{g} / \mathrm{dia}) ; \mathrm{E}_{(\mathrm{i}) \mathrm{k}}=$ efeito relativo ao experimento $\mathrm{k}$ aninhado à categoria animal i; e $\varepsilon_{\mathrm{ijk}}=$ erro aleatório, pressuposto normal e independentemente distribuído, com média zero e variância $\sigma^{2}$.

As observações relativas ao extrato etéreo aparentemente retido foram obtidas por intermédio do produto entre o consumo e o coeficiente de digestibilidade aparente.

A partir das modificações propostas para o modelo (1), descritas em (4), definiram-se as restrições para a consideração de entidade nutricional:

$0 \leq \hat{\beta}_{1}+\hat{\beta}_{2} \cdot C_{i} \leq 1$

$\hat{\alpha}_{1}+\hat{\alpha}_{2} \cdot C_{i} \leq 0$

Para validação do modelo desenvolvido a partir do coeficiente de digestibilidade verdadeiro e da contribuição metabólica fecal, foram utilizados dados oriundos de dois experimentos com vacas em lactação (Oliveira, 2005; Pina, 2005) e um com bovinos em crescimento (Silva, 2004).

Nos experimentos conduzidos por Oliveira (2005) e Pina (2005), foram utilizadas 12 vacas lactantes, distribuídas em delineamento quadrado latino 4 x 4 , com agrupamento de três quadrados, definidos conforme o tempo decorrido após o parto, totalizando 48 observações em cada experimento. Os animais foram manejados em baias individuais, em sistema de confinamento do tipo tie stall, sendo alimentados ad libitum duas vezes ao dia, logo após as ordenhas.

Os animais utilizados no experimento realizado por Oliveira (2005) foram alimentados com quatro dietas isoprotéicas (aproximadamente 14\% de PB, com base na MS), à base de silagem de milho com $40 \%$ de concentrado e 2,23\% de EE; cana-de-açúcar com $60 \%$ de concentrado e $2,18 \%$ de EE; cana-de-açúcar com $60 \%$ de concentrado e $10 \%$ de casca de café, apresentando $1,88 \%$ de EE; cana-de- 
açúcar com $60 \%$ de concentrado e $30 \%$ de casca de soja, apresentando $1,55 \%$ de EE.

Pina (2005), por sua vez, avaliou quatro dietas isoprotéicas ( $15,5 \%$ de $\mathrm{PB}$, com base na MS), com $40 \%$ de concentrado, com base na MS, diferentes quanto às fontes protéicas: farelo de soja, farelo de algodão $38 \%$, farelo de algodão $28 \%$ e uréia:sulfato de amônia $(2,26 ; 2,45 ; 1,91 \mathrm{e}$ $2,45 \%$ de EE na dieta, com base na MS, respectivamente).

No experimento conduzido por Silva (2004), foram utilizados quatro novilhos com grau de sangue predominantemente Holandês e peso médio de $320 \mathrm{~kg}$. O experimento foi dividido em cinco períodos de 15 dias, sendo os animais mantidos confinados em baias individuais, totalizando 20 observações. A dieta oferecida aos animais foi composta exclusivamente de capim-elefante, de modo que, em cada período experimental, o capim fornecido encontrava-se em um único período de rebrotação $(33,48,63,78$ ou 93 dias, apresentando, respectivamente, 1,$29 ; 1,70 ; 2,07 ; 1,54$ e $2,42 \%$ de EE, com base na MS).

Para avaliação estatística, os dados foram submetidos ao ajustamento, de forma independente aos efeitos de experimento e tratamentos, de modelo de regressão linear simples de valores observados (variável dependente) sobre valores preditos (variável independente). Os testes estatísticos foram conduzidos sob as seguintes hipóteses:

$$
\begin{array}{ll}
H_{0}: \beta_{0}=0 & H_{0}: \beta_{1}=1 \\
H_{a}: \beta_{0} \neq 0 & H_{a}: \beta_{1} \neq 1
\end{array}
$$

No caso de não-rejeição de ambas as hipóteses de nulidade, concluiu-se pela similaridade entre valores preditos e observados. Todos os procedimentos estatísticos descritos neste trabalho foram realizados por meio do programa Statistical Analysis System - SAS, adotando-se 0,05 como nível crítico de probabilidade para o erro tipo I.

A partir do banco de dados construído para validação do novo modelo, estimaram-se os teores de EE digestível utilizando-se o modelo adotado pelo NRC (2001), segundo proposições de Weiss et al. (1992):

$E E_{\text {adm }}(\%)=A G(\%)-1,4$

em que: $\mathrm{EE}_{\mathrm{adm}}(\%)=$ nível dietético de $\mathrm{EE}$ aparentemente digestível em condições de mantença, com base na MS; $\mathrm{AG}(\%)=$ nível dietético de ácidos graxos, com base na MS; e 1,4 = contribuição metabólica EE em condições de mantença.

O teor dietético de ácidos graxos foi estimado segundo a equação (Weiss et al., 1992):

$A G(\%)=E E(\%)-1 ; \forall \mathrm{EE}(\%)>1$

(8a);

$A G(\%)=0 ; \forall \mathrm{EE}(\%) \leq 1$

em que: $\mathrm{EE}(\%)$ = nível dietético de EE; sendo os demais termos definidos anteriormente.
Quando pertinente, procedeu-se à correção do teor digestível de EE predito em função do consumo a partir do teor predito de NDT, segundo porposições do NRC (2001).

A avaliação comparativa da eficiência de predição entre modelos foi realizada por meio de avaliação e partição do quadrado médio dos erros de predição (QMEP), segundo protocolos descritos por Kobayashi \& Salam (2000), conforme as equações:

$$
\begin{aligned}
& Q M E P=Q V+M a F+M o F=\frac{1}{n} \sum_{i=1}^{n}\left(x_{i}-y_{i}\right)^{2} \\
& Q V=(\bar{x}-\bar{y})^{2} \\
& M a F=\left(s_{x}-s_{y}\right)^{2} \\
& M o F=2 s_{x} s_{y}(1-r)
\end{aligned}
$$

em que: $\mathrm{x}=$ valores preditos; $\mathrm{y}=$ valores observados; QMEP = quadrado médio dos erros de predição; $\mathrm{QV}=$ quadrado do vício; $\mathrm{MaF}=$ componente relativo à magnitude de flutuação aleatória; $\mathrm{MoF}=$ componente relativo ao modelo de flutuação alatória; $\mathrm{s}_{\mathrm{x}} \mathrm{e} \mathrm{s}_{\mathrm{y}}=$ desvios-padrão para valores preditos e observados, respectivamente; e $r=$ correlação linear de Pearson entre valores preditos e observados.

Ressalta-se que, por se tratar de avaliação de erro de predição, para todos os cálculos de variâncias empregou-se como divisor o total de observações (n).

\section{Resultados e Discussão}

As bases teóricas adotadas para o desenvolvimento do modelo seguiram adaptações às proposições de Blaxter \& Mitchell (1948) e Lucas (1960), que se referem à dinâmica digestiva dos compostos nitrogenados no trato gastrintestinal de animais. Neste contexto, referindo-se ao $\mathrm{EE}$, após a ingestão e digestão, pode-se resumir a massa aparentemente não-digerida como:

$E E f=E E i+E E m+E E e$

em que: $\mathrm{EEf}=\mathrm{EE}$ fecal (g/dia); EEi = EE verdadeiramente indigerido (g/dia); EEm =EE metabólico (g/dia); e EEe =EE endógeno ( $\mathrm{g} / \mathrm{dia})$.

Define-se, portanto, como extrato etéreo metabólico a porção do EE fecal oriunda de secreções do trato digestivo (Lucas, 1960), acrescida de matéria microbiana oriunda da fermentação de componentes alimentares (Van Soest, 1994). Por outro lado, define-se como extrato etéreo endógeno a fração do EE fecal advinda da excreção celular no trato gastrintestinal (Lucas, 1960).

A partir dessas definições, relacionou-se a identidade exposta em (13) à relação ao consumo diário de EE: 


$$
\begin{aligned}
& E E c-E E f=E E c-(E E i+E E m+E E e) \\
& E E a r=E E c-E E i-E E m-E E e \\
& \frac{E E a r}{E E c}=\frac{E E c-E E i-E E m-E E e}{E E c} \\
& D a_{E E}=1-\frac{E E i}{E E c}-\frac{E E m}{E E c}-\frac{E E e}{E E c}
\end{aligned}
$$

em que: $\mathrm{EEc}=\mathrm{EE}$ consumido (g/dia); EEar = EE aparentemente retido ou digerido $(\mathrm{g} / \mathrm{dia}) ; \mathrm{Da}_{\mathrm{EE}}=$ coeficiente de digestibilidade aparente do EE; e EEf, EEi, EEm, EEe = como definidos anteriormente.

Em adaptação às proposições de Blaxter \& Mitchell (1948), pode-se afirmar que a contribuição endógena de extrato etéreo pode ser representada como uma função matemática que expressa proporcionalidade à massa metabólica do animal:

$$
\frac{E E e}{E E c}=\frac{\varepsilon \cdot P^{0,75}}{E E c}=\frac{\varepsilon}{E E c} \cdot P^{0,75}
$$

em que: $\mathrm{P}=$ massa do animal $(\mathrm{g}) ; \varepsilon=$ constante relacionada à liberação endógena de EE no trato gastrintestinal por unidade de massa metabólica $\left(\mathrm{g} / \mathrm{g} \times \mathrm{dia}^{-1}\right)$; e EEe e EEc $=$ como definidos anteriormente.

A razão ع/EEc, expressa em (15), somente poderia ser considerada significativa caso o consumo de EE assumisse valores extremamente pequenos (Lucas, 1960), possivelmente em níveis de alimentação abaixo da mantença. Assim, em condições "normais" de alimentação (mantença ou produção), ter-se-ia:

$\lim _{E E c \rightarrow E E^{\circ} C} \frac{\varepsilon}{E E c} \cdot P^{0,75}=0$

em que: $\mathrm{EE}^{\circ} \mathrm{c}=$ consumo de $\mathrm{EE}$ em condições "normais" de alimentação (g/dia); e EEc, $\varepsilon$ eP = como definidos anteriormente.

Dessa forma, rescreveu-se a equação (14d) como:

$$
\begin{aligned}
& D a_{E E}=\left(1-\frac{E E i}{E E c}\right)-\frac{E E m}{E E c} \\
& D a_{E E}=\left(1-I v_{E E}\right)-\frac{E E m}{E E c} \\
& D a_{E E}=D v_{E E}-\frac{E E m}{E E c}
\end{aligned}
$$

em que: $I v_{E E}=$ coeficiente de indigestibilidade verdadeiro do $\mathrm{EE} ; \mathrm{Dv}_{\mathrm{EE}}=$ coeficiente de digestibilidade verdadeiro do EE; e $\mathrm{Da}_{\mathrm{EE}}$, EEi, EEc e EEm = como definidos anteriormente.

Multiplicando-se ambos os termos da equação (17c) pelo consumo de EE, obteve-se:

$$
E E c \cdot D a_{E E}=E E c \cdot D v_{E E}-E E m
$$

Derivando-se (12) em função do consumo de EE, obtém-se:

$$
\frac{d\left(E E c \cdot D a_{E E}\right)}{d E E c}=D v_{E E}-\frac{d E E m}{d E E c}
$$

Assim, a diferencial expressa em (19), que representa o coeficiente de digestibilidade aparente do EE, é constituída por dois diferentes componentes: o primeiro representado pelo coeficiente de digestibilidade verdadeiro constante do EE e o segundo, por uma porção metabólica que se diferencia pela massa de EE ingerida.

Convertendo-se a equação (19) para a base percentual dietética, encontrou-se:

$E E(\%) \cdot D a_{E E}=E E(\%) \cdot D v_{E E}-C M_{E E}$

$E E_{a d}(\%)=E E_{v d}(\%)-C M_{E E}$

em que: $\mathrm{CM}_{\mathrm{EE}}=$ contribuição metabólica de EE como percentual da MS dietética; $\mathrm{EE}_{\mathrm{ad}}(\%)=$ nível dietético de EE aparentemente digestível, como percentual da MS; $\mathrm{EE}_{\mathrm{vd}}(\%)=$ nível dietético de $\mathrm{EE}$ verdadeiramente digestível, como percentual da MS; sendo os demais componentes definidos anteriormente.

Não foram observados efeitos de categoria animal sobre o coeficiente de inclinação da relação EE consumido $\mathrm{x}$ EE aparentemente retido $(\mathrm{P}>0,41)$, indicando que a digestibilidade verdadeira deste componente, representada pelo coeficiente de inclinação expresso em (4), mantém-se constante, independentemente da categoria animal. Desta forma, segundo as estimativas obtidas, obteve-se a equação:

$E E_{v d}(\%)=0,8596 \cdot E E(\%)$

em que: 0,8596 = estimativa para o coeficiente de digestibilidade verdadeiro do $\mathrm{EE}, \operatorname{com} \operatorname{IC}(\beta)_{95 \%}$ : $[0,8488 \leq \beta \leq 0,8704]$, sendo os demais componentes definidos anteriormente.

A acurácia dessa estimativa foi confirmada pela alta agregação dos pontos referentes às informações do banco de dados construído para ambas as categorias animais avaliadas $\left(\mathrm{R}^{2}=0,9983\right)$, mesmo com a adoção de um coeficiente de inclinação conjunto (Figuras 1 e 2).

A adoção do processo de estimação do teor de EE verdadeiramente digestível por meio de um coeficiente multiplicativo, como proposto neste estudo, contrapõe-se às proposições de Weiss et al. (1992) e do NRC (2001), baseadas na estimação por subtração de constante. Contudo, as diferenças entre valores observados e estimados por este sistema em condições nacionais (Rocha Jr. et al., 2003; Silva, 2004; Campos, 2004; Oliveira, 2005) evidenciaram, inicialmente, a inacurácia dessa proposição para sua aplicação em estudos com alimentos produzidos em condições tropicais.

A adoção de um desconto por subtração de constante (Weiss et al., 1992; NRC, 2001) implica que toda e qualquer 


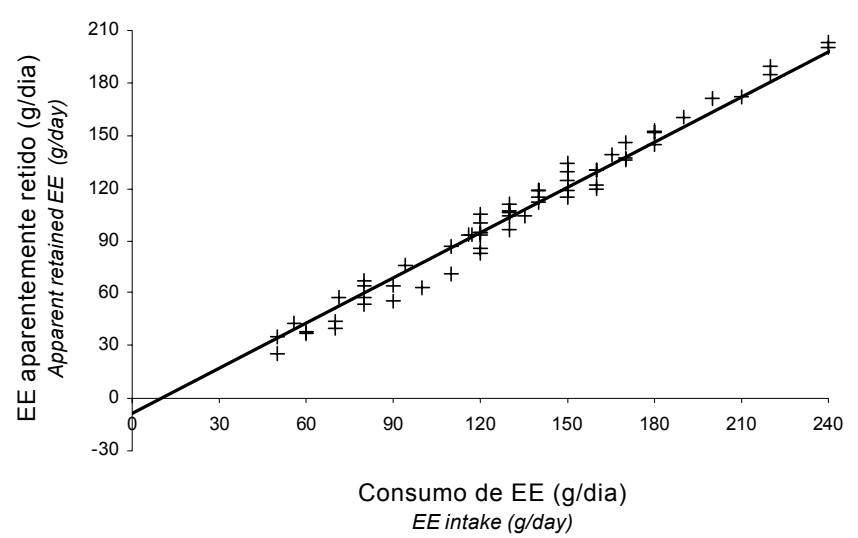

Figura 1 - Relação consumo x quantidade aparentemente retida de EE em bovinos em crescimento e terminação (a linha de tendência corresponde à reta de quadrados mínimos).

Figure 1 - Relationship between intake and EE apparently retained in growing and finishing cattle (the tendency line corresponds to least squares straight line).

fonte de EE, à exceção de fontes lipídicas (não-contempladas no banco de dados deste estudo), possui fração absoluta comum de compostos não-inseríveis no metabolismo animal, a qual representaria o complemento da quantidade de ácidos graxos presentes no alimento, normalmente representado por ceras, carotenóides e outros compostos indigestíveis (Van Soest, 1994).

Contudo, a inspeção de dados nos quais se relacionam os teores de EE e ácidos graxos (Sukhija \& Palmiquist, 1988; Van Soest, 1994) evidenciou elevada variabilidade dessa relação entre alimentos, que pode implicar distorções sobre as estimativas de teor digestível a partir da subtração de uma constante. Desta forma, a pressuposição de proporcionalidade entre o material digestível e o nãodigestível levaria à compensação entre fontes com diferentes teores de EE, tornando mais adequada a adoção de correções com base em fatores multiplicativos, como adotado neste trabalho. A eficiência desta proposição, ao menos em termos de construção de modelo, pôde ser confirmada pelas relações expressas nas Figuras 1 e 2.

Em contrapartida à similaridade observada para o coeficiente de digestibilidade verdadeiro, verificou-se efeito significativo $(\mathrm{P}<0,02)$ de categoria animal sobre a excreção de EE metabólico, cujas estimativas obtidas diretamente pelo modelo descrito em (4) foram de 8,36 e 35,66 g/dia, para bovinos em crescimento e terminação e vacas em lactação, respectivamente (Figuras 1 e 2). Ressalta-se que as estimativas obtidas para o coeficiente de digestibilidade verdadeiro e as contribuições metabólicas enquadraram-se nas restrições impostas em (5) e (6), garantindo o status de

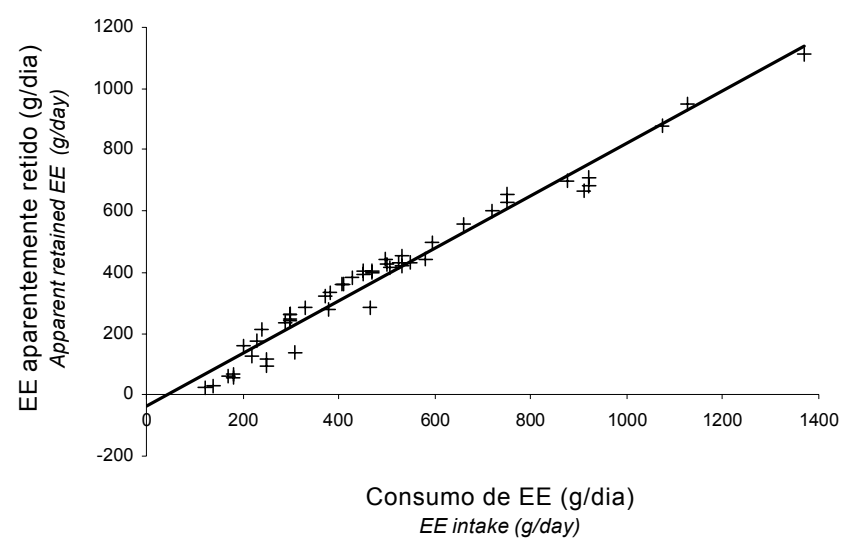

Figura 2 - Relação consumo x quantidade aparentemente retida de EE em vacas em lactação (a linha de tendência corresponde à reta de quadrados mínimos).

Figure 2 - Relationship between intake and EE apparently retained in lactating cows (the tendency line corresponds to least squares straight line).

entidade nutricional ao EE (Lucas \& Smart, 1959) e suportando os métodos de estimação adotados neste trabalho.

A superioridade da fração metabólica do EE em vacas em lactação, em comparação a animais em crescimento e terminação $(\mathrm{P}<0,02)$ (Figuras 1 e 2 ) pode ser suportada por sua relação diretamente proporcional ao consumo de EE (Lucas, 1960) (Tabela 1).

Por outro lado, a constatação de um coeficiente de digestibilidade verdadeiro constante e independente da categoria animal, associado à contribuição metabólica diferenciada em função do consumo de EE, converge com exatidão às pressuposições teóricas sumariadas na equação (19), garantindo a validade do modelo proposto neste trabalho.

Com vistas à estimação do teor de EE aparentemente digestível (parâmetro base para o modelo proposto neste trabalho), o qual constitui componente direto do teor de NDT da dieta, procedeu-se à conversão das estimativas de EE metabólico (g/dia) em função do teor de EE dietético para cada valor integrante do banco de dados, utilizando-se a equação:

$$
\begin{aligned}
& E E_{m}(\%)=\frac{E E_{v d}-E E a r}{E E c} \cdot E E(\%) \\
& E E_{m}(\%)=\frac{0,8596 \cdot E E c-D a_{E E} \cdot E E c}{E E c} \cdot E E(\%) \\
& E E_{m}(\%)=\left(0,8596-D a_{E E}\right) \cdot E E(\%)
\end{aligned}
$$

em que: $\mathrm{EE}_{\mathrm{m}}(\%)=\mathrm{EE}$ metabólico, como percentual da MS dietética; sendo os demais componentes definidos anteriormente. 
A avaliação dos resultados produzidos via equação (22c) para cada categoria animal indicou não haver relação entre a contribuição metabólica percentual e o nível dietético de $E E(P>0,71)$, permitindo-se, desta forma, a representação dessa fração por valores constantes:

$E E_{a d}(\%)=0,8596 \cdot E E(\%)-0,18 \quad($ Bovinos em crescimento e terminação)

$$
E E_{a d}(\%)=0,8596 \cdot E E(\%)-0,21 \text { (Vacas em lactação) }
$$

em que: 0,18 e 0,21 = estimativas para o EE metabólico, como percentual da MS da dieta; sendo os demais termos definidos anteriormente.

Considerando as características do banco de dados utilizado neste estudo, ressalta-se que as estimativas obtidas a partir das equações (23a) e (23b) são aplicáveis a animais submetidos a consumo ad libitum, ou seja, em nível de produção. Segundo observações realizadas em condições brasileiras, a relação entre o nível de NDT em situações de produção e mantença (consumo restrito) aproxima-se de 0,95 (Costa et al., 2005), que, possivelmente, se projeta para cada um de seus componentes.

Segundo as relações obtidas neste trabalho (Figuras 1 e 2), não se esperam alterações no coeficiente de digestibilidade verdadeiro do EE, em função do nível de consumo - diferenciação observada somente sobre a contribuição metabólica fecal (Equação 19). Portanto, tomando-se como base o consumo médio de EE observado para cada categoria (Tabela 1) e a relação de digestibilidade nos níveis de produção e mantença relatada por Costa et al. (2005), obtêm-se as estimativas do teor dietético de EE aparentemente digerido em condições de mantença $\left(\mathrm{EE}_{\mathrm{adm}}\right)$ por:

$E E_{a d m}(\%)=0,8596 \cdot E E(\%)-0,09$ (bovinos em crescimento e terminação)

$E E_{\text {adm }}(\%)=0,8596 \cdot E E(\%)-0,10($ vacas em lactação $)$

As estimativas dos parâmetros de regressão associadas aos respectivos níveis descritivos de probabilidade para os testes de hipóteses são apresentadas na Tabela 2.

Verificou-se a não-rejeição de ambas as hipóteses de nulidade ( $\mathrm{P}>0$,29) (Tabela 2 e Figura 3), comprovando efetivamente a adequação do modelo proposto (Equação 19) e das equações estabelecidas para as categorias animais avaliadas (23a e 23b).

Embora a inspeção gráfica do comportamento dos dados (Figura 3) tenha evidenciado certo nível de variação dos dados em torno da linha de equalidade ( $\mathrm{Y}=\mathrm{X})$, este nível não causou discrepância significativa entre valores preditos e observados ( $\mathrm{P}>0,29)$ (Tabela 2). Entende-se que esse resultado advenha de variações no consumo total de EE inerentes a cada animal, o que resulta diretamente em variações na porção metabólica fecal do EE (Equação 19). Como as equações propostas foram baseadas em valores médios (23a e 23b), cujo conjunto de dados utilizado para estimação não evidenciou relações $(\mathrm{P}>0,71)$ com o nível dietético de EE, essas variações podem ser consideradas pouco relevantes e não comprometedoras ao processo de estimação.

Tabela 2 - Estimativas dos parâmetros da regressão, níveis descritivos de probabilidade para o erro tipo I e desvio-padrão residual $\left(s_{X Y}\right)$ para as relações entre os valores preditos (variável independente) e os observados (variável dependentes) para os níveis dietéticos de EE aparentemente digestível

Table 2- Estimates of regression parameters, descriptive levels of probability for type I error, and residual standard deviation $\left(s_{X Y}\right)$ for the relationship between predicted (independent variable) and observed (dependent variable) for dietary levels of ether extract apparently digestible

\begin{tabular}{lcc}
\hline $\begin{array}{l}\text { Parâmetro } \\
\text { Parameter }\end{array}$ & $\begin{array}{c}\text { Estimativa } \\
\text { Estimate }\end{array}$ & $\begin{array}{c}\text { Valor-P } \\
P \text { value }\end{array}$ \\
\hline $\begin{array}{l}\text { Intercepto }^{1} \\
\text { Intercept }\end{array}$ & 0,0799 & 0,2947 \\
Coeficiente de inclinação $^{2}$ & 1,0168 & 0,7252 \\
Slope coefficient & & \\
$\mathrm{s}_{\mathrm{xy}}$ & & 0,1402 \\
$\mathrm{n}$ & \multicolumn{2}{c}{116} \\
\hline${ }^{1} \mathrm{H}_{0}: \beta_{0}=0 ; \mathrm{H}_{\mathrm{a}}: \beta_{0} \neq 0$. & & \\
${ }^{2} \mathrm{H}_{0}: \beta_{1}=1 ; \mathrm{H}_{\mathrm{a}}: \beta_{1} \neq 1$. & &
\end{tabular}

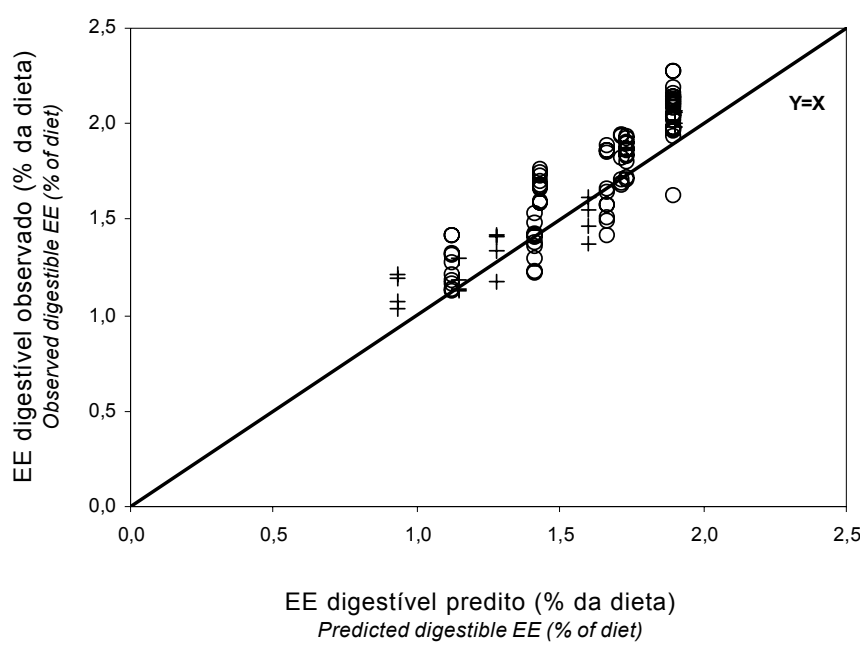

Figura 3 - Relação entre valores preditos e observados para os teores de EE digestível na dieta $(+=$ bovinos em crescimento e terminação; o = vacas em lactação).

Figure 3 - Relationship between predicted and observed values for digestible EE (+ = growing and finishing cattle; $o$ = lactating cows). 
Verificou-se, porém, baixa amplitude do erro de predição e de seus componentes para os teores dietéticos de EE aparentemente digestível obtidos pelo modelo proposto neste trabalho (Tabela 3 ). Em contrapartida, elevados erros de predição foram obtidos pela aplicação do modelo atualmente adotado pelo sistema NRC (2001) (Tabela 3). Pela estrutura básica deste modelo (Equações 7, 8 a e 8 b), valores de EE dietético inferiores a 2,4\% conduzem a estimativas negativas do teor de EE digestível, o que conduz a estimativas inverossímeis frente à real fração digestível de EE observada em condições tropicais, cujos teores dietéticos de EE situam-se, comumente, em patamares inferiores a 2,4\% da MS total (Campos, 2004; Silva, 2004; Oliveira, 2005; Pina, 2005).

Embora ambos os modelos tenham apresentado pequenas limitações quanto à magnitude e ao modelo de

Tabela 3 - Médias, desvios-padrão (s), coeficiente de correlação linear de Pearson (r), erro médio de predição (EMP), quadrado médio do erro de predição (QMEP), quadrado do vício (QV), componente de magnitude de flutuação aleatória (MaF) e componente de modelo de flutuação aleatória (MoF) para os teores dietéticos de EE digestível (\% da MS dietética) $(n=116)$

Table 3 - Means, standard deviation (s), Pearson linear correlation ( $r$ ), mean prediction error (MPE), mean square of prediction error (MSPE), square of bias (SB), component for magnitude of random fluctuation (MaF), and component for model of random fluctuation (MoF) for dietary levels of ether extract apparently digestible (\% of dry matter) $(n=116)$

\begin{tabular}{|c|c|c|c|c|}
\hline \multirow[b]{2}{*}{$\begin{array}{l}\text { Item } \\
\text { Item }\end{array}$} & \multirow[t]{2}{*}{$\begin{array}{c}\text { Valor observado } \\
\text { Observed value }\end{array}$} & \multicolumn{3}{|c|}{$\begin{array}{l}\text { Valor predito } \\
\text { Predicted value }^{1}\end{array}$} \\
\hline & & $\begin{array}{l}\text { Modelo NRC I } \\
\text { NRC model I }\end{array}$ & $\begin{array}{l}\text { Modelo NRC II } \\
\text { NRC model II }\end{array}$ & $\begin{array}{l}\text { Novo modelo } \\
\text { Proposed model }\end{array}$ \\
\hline Média (Mean) & 1,67 & $-0,34$ & 0,01 & 1,57 \\
\hline $\mathrm{s}$ & 0,33 & 0,34 & 0,02 & 0,29 \\
\hline$r^{2}$ & - & 0,9066 & 0,6870 & 0,9027 \\
\hline EMP (MPE) & - & $-2,01$ & $-1,66$ & $-0,10$ \\
\hline QMEP (MSPE) & - & 4,07 & 2,86 & 0,03 \\
\hline QV (SB) & - & 4,05 & 2,76 & 0,01 \\
\hline $\mathrm{MaF}$ & - & 0,00 & 0,09 & 0,00 \\
\hline $\mathrm{MoF}$ & - & 0,02 & 0,00 & 0,02 \\
\hline
\end{tabular}

${ }^{1}$ Modelo NRC I: valores aritméticos obtidos via modelo NRC; Modelo NRC II: desconsiderando-se os valores negativos produzidos pelo modelo, considerados equivalentes a 0 (NRC Model I: it was considered the arithmetic values obtained by the NRC model; NRC Model II: the negative values were considered as zero).

2 Todas os valores de correlação foram significativamente diferentes de $0(P<0,01)$ [All correlation values were significantly different from zero $(P<0.01)$ ]

flutuação dos desvios em torno da média aritmética (Tabela 3), a maior limitação observada para o modelo adotado pelo sistema NRC (2001) reside na dimensão do vício, que permaneceu significativamente elevado mesmo após a interpretação dos resultados negativos produzidos como "zero" (Tabela 3). Desta forma, a correção por subtração de constante (Weiss et al., 1992) parece constituir empecilho à correta estimação da fração verdadeiramente digestível do EE frente à elevada variação da fração de ácidos graxos nos alimentos, justificando novamente a melhor adequação de um termo multiplicativo (Equação 21) para estimação dessa porção.

Segundo descrições de Weiss et al. (1992), observarse-ia fração metabólica constante de EE, independentemente da categoria animal avaliada (Equação 7). Esse pressuposto pode estar incrementando o vício observado no modelo adotado pelo sistema NRC (2001), pois, conforme demonstrado neste trabalho, tanto em termos teóricos (Equação 19) como empíricos (Equações 23a e 23 b), verificam-se divergências para esta fração em função da categoria animal avaliada, o que constitui reflexo direto do nível de consumo observado (Equação 19).
A acurácia obtida pelo modelo proposto neste trabalho, tanto em termos diretos (Tabela 2) como em termos comparativos ao modelo adotado pelo NRC (2001) (Tabela 3), evidenciou sua capacidade preditiva do teor dietético de EE digestível em alimentos produzidos em condições tropicais, o que permite sugerir sua incorporação ao sistema de equações somativas adotado pelo NRC (2001) com vistas à obtenção de maior exatidão nas estimativas do teor de NDT a partir da composição química dos alimentos.

\section{Conclusões}

A estimação da fração digestível do EE dietético sob os pressupostos de constância no coeficiente de digestibilidade verdadeiro e de diferenciação da contribuição metabólica em função do nível de consumo mostra-se acurada para alimentos/dietas produzidos em condições tropicais.

Os modelos propostos neste estudo poderão ampliar a acurácia do processo de predição energética via composição química, se incorporados ao sistema de equações somativas adotado pelo sistema nutricional NRC (2001). 


\section{Literatura Citada}

ASSIS, A.J.; CAMPOS, J.M.S.; VALADARES FILHO, S.C. et al. Polpa cítrica em dietas de vacas em lactação. 1.Consumo de nutrientes, produção e composição do leite. Revista Brasileira de Zootecnia, v.33, n.1, p.242-250, 2004a.

ASSIS, A.J.; CAMPOS, J.M.S.; QUEIROZ, A.C. et al. Polpa cítrica em dietas de vacas em lactação. 2.Digestibilidade dos nutrientes em dois períodos de coleta de fezes, $\mathrm{pH}$ e nitrogênio amoniacal do líquido ruminal. Revista Brasileira de Zootecnia, v.33, n.1, p.251-257, 2004b.

ASSOCIATION OF OFFICIAL ANALYTICAL CHEMISTRY AOAC. Official methods of analysis. 15.ed. Arlington: AOAC International, 1990. 1117p.

BLAXTER, K.L.; MITCHELL, H.H. The factorization of the protein requeriments of ruminants and of the protein values of feeds, with particular reference to the significance of metabolic fecal nitrogen. Journal of Animal Science, v.7, n.2, p.351372, 1948.

CAMPOS, P.R.S.S. Estimativas do valor energético de alguns volumosos e da digestibilidade da fibra em detergente neutro em ovinos. Viçosa, MG: Universidade Federal de Viçosa, 2004. 67p. Dissertação (Mestrado em Zootecnia) - Universidade Federal de Viçosa, 2004.

CARDOSO, R.C.; VALADARES FILHO, S.C.; COELHO DA SILVA, J.F. et al. Consumo e digestibilidades aparentes totais e parciais de rações contendo diferentes níveis de concentrado, em novilhos F1 Limousin x Nelore. Revista Brasileira de Zootecnia, v.39, n.6, p.1832-1843, 2000.

CARVALHO, A.U.; VALADARES FILHO, S.C.; COELHO DA SILVA, J.F. et al. Níveis de concentrado na dieta de zebuínos. 1. Consumo e digestibilidade aparente. Revista Brasileira de Zootecnia, v.26, n.5, p.986-995, 1997.

CHIZZOTTI, M.L. Avaliação da casca de algodão para novilhos de origem leiteira e determinação da excreção de cretinina e produção de proteína microbiana em novilhas e vacas. Viçosa, MG: Universidade Federal de Viçosa, 2004. 132p. Dissertação (Mestrado em Zootecnia) - Universidade Federal de Viçosa, 2004.

CHIZZOTTI, M.L.; VALADARES FILHO, S.C.; LEÃO, M.I. et al. Casca de algodão em substituição parcial à silagem de capim-elefante para novilhos. 1. Consumo, degradabilidade e digestiblidades total e parcial. Revista Brasileira de Zootecnia, v.34, n.6, p.2093-2102, 2005.

CONRAD, H.R.; WEISS, W.P.; ODWONGO, W.O. et al. Estimating net energy lactation from components of cell solubles and cell walls. Journal of Dairy Science, v.67, n.2, p.427-436, 1984.

COSTA, M.A.L; VALADARES FILHO, S.C.; VALADARES, R.F.D. et al. Validação das equações do NRC (2001) para predição do valor energético de alimentos em condições brasileiras. Revista Brasileira de Zootecnia, v.34, n.1, p.280-287, 2005.

DETMANN, E.; ZERVOUDAKIS, J.T.; CABRAL, L.S. et al. Validação de equações preditivas da fração indigestível da fibra em detergente neutro em gramíneas tropicais. Revista Brasileira de Zootecnia, v.33, n.6, p.1866-1875, 2004 (supl. 1).

DETMANN, E.; PAULINO, M.F.; CABRAL, L.S. et al. Simulação e validação de parâmetros da cinética digestiva em novilhos mestiços suplementados a pasto por intermédio de sistema in vitro de produção de gases. Revista Brasileira de Zootecnia, v.34, n.6, p.2112-2122, 2005.

DIAS, H.C.; VALADARES FILHO, S.C.; COELHO DA SILVA, J.F. et al. Consumo e digestões totais e parciais em novilhos F1 limousin $\mathrm{x}$ nelore alimentados com dietas contendo cinco níveis de concentrado. Revista Brasileira de Zootecnia, v.29, n.2, p.545-554, 2000.

DRAPER, N.; SMITH, H. Applied regression analysis. New York: John Willey \& Sons, 1966. 407p.
ÍTAVO, L.C.V.; VALADARES FILHO, S.C.; SILVA, F.F. et al. Níveis de concentrado e proteína bruta na dieta de bovinos nelore nas fase de recria e terminação: consumo e digestibilidade. Revista Brasileira de Zootecnia, v.31, n.2, p.1033-1041, 2002a (supl.).

ÍTAVO, L.C.V.; VALADARES FILHO, S.C.; SILVA, F.F. et al. Consumo e digestibilidades aparentes totais e parciais de nutrientes em novilhos alimentados com dietas contendo vários níveis de concentrado. Revista Brasileira de Zootecnia, v. 31 , n.3, p.1543-1552, 2002b (supl.).

LADEIRA, M.M.; VALADARES FILHO, S.C.; COELHO DA SILVA, J.F. et al. Consumo e digestibilidades aparentes totais e parciais de dietas contendo diferentes níveis de concentrado em novilhos nelore. Revista Brasileira de Zootecnia, v.28, n.2, p.395403, 1999.

LUCAS, H.L. Relations between apparent digestibility and the composition of feed and feces. 1. A quantitative theory. Raleigh: North Caroline State College, 1960. 55p. (Technical Report).

LUCAS, H.L.; SMART, W.W.G. Chemical composition and the digestibility of forages. In: PASTURE AND CROP IMPROVEMENT CONFERENCE, 16., 1959, Mississipi. Proceedings... Mississipi: Mississipi State University, 1959. p.23-26.

MAGALHÃES, M.C.S.; VÉRAS, A.S.C.; FERREIRA, M.A. et al. Inclusão de cama de frango em dietas à base de palma forrageira (Opiuntia ficusindica Mill) para vacas mestiças em lactação. 1.Consumo e produção. Revista Brasileira de Zootecnia, v.33, n.6, p.1897-1908, 2004a (sup1. 1).

MAGALHÃES, M.C.S.; VÉRAS, A.S.C.; CARVALHO, F.F.R. et al. Inclusão de cama de frango em dietas à base de palma forrageira (Opiuntia ficusindica Mill) para vacas mestiças em lactação. 2. Digestibilidade aparente. Revista Brasileira de Zootecnia, v.33, n.6, p.1909-1919, 2004b (supl. 1).

MALAFAIA, P.A.M.; VALADARES FILHO, S.C.; COELHO DA SILVA, J.F. et al. Sebo bovino em rações para vacas em lactação. 1. Consumo dos nutrientes, produção e composição do leite. Revista da Sociedade Brasileira de Zootecnia, v.25, n.1, p.153-163, 1996a.

MALAFAIA, P.A.M.; VALADARES FILHO, S.C.; COELHO DA SILVA, J.F. et al. Sebo bovino em rações para vacas em lactação. 2. Digestão total e parcial dos alimentos. Revista da Sociedade Brasileira de Zootecnia, v.25, n.1, p.164-176, 1996b.

MENDONÇA, S.S.; CAMPOS, J.M.S.; VALADARES FILHO, S.C. et al. Consumo, digestibilidade aparente, produção e composição do leite e variáveis ruminais em vacas leiteiras alimentadas com dietas à base de cana-de-açúcar. Revista Brasileira de Zootecnia, v.33, n.2, p.481-492, 2004.

MORA, P.J.G.; VALADARES FILHO, S.C.; LEÃO, M.I. et al. Digestibilidade aparente dos nutrientes e energia líquida da silagem de milho (Zea mays L.) para vacas lactantes. Revista da Sociedade Brasileira de Zootecnia, v.25, p.357-368, 1996 a.

MORA, P.J.G.; LEÃO, M.I.; VALADARES FILHO, S.C. et al. Grão de soja em rações para vacas lactantes: consumo de nutrientes, produção e composição de leite. Revista da Sociedade Brasileira de Zootecnia, v.25, p.369-381, 1996b.

MOREIRA, A.L.; PEREIRA, O.G.; GARCIA, R. et al. Produção de leite, consumo e digestibilidade aparente dos nutrientes, $\mathrm{pH}$ e concentração de amônia ruminal em vacas lactantes recebendo rações contendo silagem de milho e fenos de alfafa e e capimcoastcross. Revista Brasileira de Zootecnia, v.30, n.3, p.1089-1098, 2001 (supl. 1).

NATIONAL RESEARCH COUNCIL - NRC. Nutrient requirements of dairy cattle. 7.ed. Washington: Academic Press, 2001. 381p.

NÖRNBERG, J.L.; STUMPF JR., W.; LÓPEZ, J. et al. Valor do farelo de arroz integral como fonte de gordura na dieta de vacas jersey na fase inicial de lactação: digestibilidade aparente dos nutrientes. Revista Brasileira de Zootecnia, v.33, n.6, p.2412-2421, 2004 (supl. 3). 
OLIVEIRA, A.S.; VALADARES, R.F.D.; VALADARES FILHO, S.C. et al. Consumo, digestibilidade aparente e composição do leite em vacas alimentadas com quatro níveis de compostos nitrogenados não-protéicos. Revista Brasileira de Zootecnia, v.30, n.4, p.1358-1366, 2001.

OLIVEIRA, A.S. Casca de café ou casca de soja em substituição ao milho em dietas à base de cana-de-açúcar para vacas leiteiras. Viçosa, MG: Universidade Federal de Viçosa, 2005, 90p. Dissertação (Mestrado em Zootecnia) - Universidade Federal de Viçosa, 2005.

PINA, D.S. Fontes de proteína para vacas em lactação. Viçosa, MG: Universidade Federal de Viçosa, 2005. 84p. Dissertação (Mestrado em Zootecnia) - Universidade Federal de Viçosa, 2005.

RENNÓ, L.N. Consumo, digestibilidade total e parcial, produção microbiana, parâmetros ruminais e excreções de uréia e creatinina em novilhos alimentados co dietas contendo quatro níveis de uréia ou dois níveis de proteína. Viçosa, MG: Universidade Federal de Viçosa, 2003, 267p. Tese (Doutorado em Zootecnia) - Universidade Federal de Viçosa, 2003.

ROCHA JR., V.R.; VALADARES FILHO, S.C.; BORGES, A.M. et al. Estimativa do valor energético dos alimentos e validação das equações propostas pelo NRC (2001). Revista Brasileira de Zootecnia, v.32, n.2, p.480-490, 2003.

SCHOFIELD, P.; PITT, R.E.; PELL, A.N. Kinetics of fiber digestion from in vitro gas production. Journal of Animal Science, v.72, n.11, p.2980-2991, 1994

SILVA, P.A. Valor energético do capim-elefante em diferentes idades de rebrota e estimativa da digestibilidade in vivo da fibra em detergente neutro. Viçosa, MG: Universidade Federal de Viçosa, 2004. 72p. Dissertação (Mestrado em Zootecnia) - Universidade Federal de Viçosa, 2004.

SILVA, R.M.N.; VALADARES, R.F.D.; VALADARES FILHO, S.C. et al. Uréia para vacas em lactação. 1.Consumo, digestibilidade, produção e composição do leite. Revista Brasileira de Zootecnia, v.30, n.5, p.1639-1649, 2001.
SUKHIJA, P.S.; PALMQUIST, D.L. Rapid method for determination of total fatty acid content and composition of feedstuffs and feces. Journal of Agricultural and Food Chemistry, v. 36 , p.1202-1206, 1988.

SOARES, C.A.; CAMPOS, J.M.S.; VALADARES FILHO, S.C. et al. Consumo, digestibilidade, produção e composição do leite de vacas leiteiras alimentadas com farelo de trigo. Revista Brasileira de Zootecnia, v.33, n.6, p.2161-2169, 2004 (sup1. 2).

TIBO, G.C.; VALADARES FILHO, S.C.; VALADARES, R.F.D. et al. Níveis de concentrado em dietas de novilhos mestiços F1 Simental x Nelore. 1. Consumo e digestibilidades. Revista Brasileira de Zootecnia, v.29, n.3, p.910-920, 2000.

TILLEY, J.M.A.; TERRY, R.A. A two-stage technique for the in vitro digestion of forage crops. Journal of British Grassland Society, v.18, n.2, p.104-111, 1963.

Van SOEST, P.J. Nutritional ecology of the ruminant. Ithaca: Cornell University Press, 1994. 476p.

VIEIRA, R.A.M.; PEREIRA, J.C.; MALAFAIA, P.A.M. et al. Simulação da dinâmica de nutrientes no trato gastrintestinal: aplicação e validação de um modelo matemático para bovinos a pasto. Revista Brasileira de Zootecnia, v.29, n.3, p.898909, 2000.

WEISS, W.P.; CONRAD, H.R.; St.PIERRE, N.R. A theroticallybased model for predicting total digestible nutrient values of forage and concentrates. Animal Feed Science and Technology, v.39, p.95-110, 1992. 